Results: Our study population comprising 45 females and 15 males had mean age $( \pm S D)$ of 23.55 years $( \pm 5.55$ years). Two-thirds of patients had mild to very severe depression. Majority (44 patients) of the patients had mild anxiety and poor quality of sleep. QOLIE-31 score was higher among males, those with education level above 12th standard and belonging to middle and upper socioeconomic groups. Patients with myoclonic seizures alone had better quality of life compared with patients with GTCS-absence semiology $(p<0.05)$. QoL negatively correlated with severity of depressive and anxiety symptomatology and quality of sleep. Statistically significant correlation between duration of medication and per capita income with severity of depressive and anxiety symptoms was observed. Multivariate analysis did not show any significant correlation of demographic and clinical factors with QoL.

Conclusion: Clinicodemographic factors intricately affect quality of life in patients with JME. Poor socioeconomic class and presence of comorbid depression, anxiety, and poor sleep quality are associated with poorer quality of life among patients with JME.

\section{A0035: The Study of Cognitive Functions and Neuropsychiatric Comorbidities among Intractable Epilepsy Patients in a Tertiary Care Hospital R.M. Bhoopathy, ${ }^{1}$ E. Uma maheswari, ${ }^{1}$ A.V. Srinivasan ${ }^{1}$ 'Tamil Nadu Government Multi Super Speciality Hospital, Chennai, Tamil Nadu, India}

Introduction: People suffering with epilepsy often present with cognitive dysfunction, neuropsychiatric comorbidities, such as cognitive impairment, depression, anxiety, and other behavioral problems. These illnesses may aggravate the epileptic manifestations in intractable epilepsy patients. The cognitive dysfunction and psychiatric comorbidities may be due to the intractable seizure itself, structural damage to the brain, and antiepileptic drugs. There is lack of studies in Indian context regarding the cognitive functions and neuropsychiatric comorbidities in intractable epilepsy patients.

Aim: To study the cognitive functions (IQ MQ visuoperceptual functions) and neuropsychiatric comorbidities (anxiety and depression) in intractable epilepsy patients.

Methods: Around 600 patients who fulfilled the criteria of drug-resistant epilepsy (ILAE, 2010) were included in the study. Only 506 patients underwent detailed history and neurological examination, radiological investigations, neuropsychological evaluation, educational, occupational status assessment, and quality of life assessment. Neuropsychological evaluations (intelligent quotient [IQ], the mental quotient $[\mathrm{MQ}]$, Bender-Gestalt test [BGT], anxiety and depression scales) were performed within 2 to 3 months after the radiological investigation.

Results: Out of 506 patients with intractable epilepsy, 147 patients $(29.05 \%)$ had mental retardation $(<69)$ score in Wechsler intelligence scale. $20 \%(n=104)$ of them had dull normal intelligence. Two hundred and fifty-five patients (50\%) of them had average intelligence. Wechsler's memory quotient scores were low $(<70)$ in 194 patients $(38.34 \%)$ and showed memory dysfunction. BGT revealed abnormal visuoperceptual gestalt functions in 218 patients (43.08\%). Multiphasic personality questionnaire to assess anxiety and depression could not be administered in 147 patients who had mental retardation. Multiphasic personality questionnaire administered to patients without mental retardation showed anxiety in 161 patients (31.82\%), depression in 142 patients (28.06\%), and mixed anxiety and depression in 126 patients (24.90\%). Pearson's Chi-square test did not reveal any significant difference between the IQ MQ BGT, anxiety, depression and quality of life scores between the generalized and partial seizures. Abnormal IQ scores (below average), MQ scores, BGT Results were strongly associated $(p<0.01)$ with MTS. Significant proportion of patients with intractable epilepsy who had no structural abnormalities had normal IQ scores, MQ scores, and BGT Results. A significant proportion of patients with MTS had anxiety and depression. Also, a significant proportion of patients without any structural abnormalities did not show any signs of anxiety or depression. Another significant proportion of patients with MTS had combined symptoms of anxiety and depression $(p<0.05)$.

Conclusion: The present study highlights that patients with intractable epilepsy are often associated with neuropsychological manifestations, such as cognitive impairment (mental retardation: 29.05\%, memory impairment: $38.34 \%$, abnormal visuoperceptual gestalt functions: $43.08 \%$ ), anxiety (31.82\%), depression (28.06\%), and mixed anxietydepression (24.90\%). Hence, diagnosing these conditions is very important among the intractable epilepsy patient and treating them effectively with drugs as well as counseling.

A0036: Effect of T-Type of Calcium Channel Blockers on Behavioral, Biochemical, Immunohistochemical, Oxidative, and Histopathological Parameters in Chemically Induced Seizure Tests in Wistar Albino Rats Saniya K.,' Patil B.G.,' Madhavrao C, ${ }^{2}$ Prakash K.G., ${ }^{1}$ Mythili Bai K. ${ }^{3}$

'Department of Anatomy, All India Institute of Medical Sciences, Delhi, India

${ }^{2}$ Department of Pharmacology, All India Institute of Medical Sciences, Delhi, India

${ }^{3}$ Department of Physiology, All India Institute of Medical Sciences, Delhi, India

Objective: To study the effect of t-type of calcium channel blockers on behavioral, biochemical, immunohistochemical, oxidative, and histopathological parameters in chemically induced seizure tests in Wistar albino rats.

Methods: The study proposal was approved by the Institutional Review Board (IRB) and all study procedures were performed in accordance with the CPCSEA guidelines. The study was done on the healthy, adult Wistar albino rats of either sex as per the standard protocol described in the literature. This study consisted of four groups, each with six animals. The study groups were group I (vehicle control), group II (negative control), group III (positive control), and group IV (experimental drug [t-type of calcium channel blocker: Flunarizine]). Statistical software, GraphPad Instat 3.0 version 